Chirurg 2013 $\cdot 84: 328$

DOI 10.1007/s00104-013-2491-2

Online publiziert: 10. März 2013

๑) Springer-Verlag Berlin Heidelberg 2013

L. Haeder $\cdot J$. Jähne

Klinik für Allgemein- und Viszeralchirurgie, Schwerpunkt für endokrine und onkologische Chirurgie,

Diakoniekrankenhaus Henriettenstiftung gGmbH, Hannover

\title{
Präoperative Stagingverfahren beim Magenkarzinom
}

führt, die gefilterten Artikel selektioniert sowie alle die Einschlusskriterien erfüllenden Publikationen ( $\mathrm{n}=21 / 5204, \mathrm{n}=22 / 7117$, $\mathrm{n}=28 / 1129$ ) nach unabhängiger Sichtung extrahiert. Die Auswertung erfolgte anschließend mittels statistischer Analysen hinsichtlich Genauigkeit, Sensitivität und Spezifität.

\section{Ergebnisse}

Für das T-Staging zeigte die Magnetresonanztomographie (MRT) - bei allerdings geringerer Patientenzahl - bessere Ergebnisse als die Computertomographie (CT; Gesamtgenauigkeit $83 \%$ vs. $71,5 \%$ ). Für das N-Staging ergaben sich kaum signifikante Unterschiede mit der niedrigsten Sensitivität (40\%), aber zugleich auch höchsten Spezifität (98\%) für die PET(Positronenemissionstomographie)/ CT. Die Detektion von Fernmetastasen lieferte beim Multidetektor-CT (82\%) und beim PET/CT (88\%) die höchste Genauigkeit, wobei hier der Wert des MRT nicht evaluiert wurde.

Die diagnostische Genauigkeit des EUS lag für das T-Staging im Mittel bei 75\% mit besseren Ergebnissen bei fortgeschrittenen Stadien (T3/4). Hinsichtlich des $\mathrm{N}$-Stagings ergab sich eine mittlere Trefferquote von $64 \%$ (Sensitivität $74 \%$, Spezifität $80 \%$ ), wobei sich eine signifikante und nicht erklärbare Heterogenität der eingeschlossenen Studien fand.

Trotz einer eingeschränkten Beurteilbarkeit des 3. Reviews hinsichtlich der Interpretation des Nachweises freier intraperitonealer Tumorzellen (IFCCs) scheint hier eine prognostische Wertigkeit vorzuliegen. Der positive Nachweis ist demnach mit dem erhöhten Risiko eines peritonealen Tumorrezidives (11,1-100\%) so- wie einem signifikant geringeren Gesamtüberleben assoziiert, wobei auch bei IFCC-negativem Status Peritonealkarzinoseraten von $0-51 \%$ nachgewiesen werden konnten.

\section{Fazit}

Obwohl mit den bildgebenden Verfahren eine Verbesserung des prätherapeutischen Stagings beim Magenkarzinom erreicht werden konnte, ist die Sensitivität der einzelnen Modalitäten nicht optimal und insbesondere bei der (Endo-) Sonographie untersucherabhängig, sodass eine Kombination diagnostischer Verfahren angestrebt werden sollte, um den prädiktiven Wert zu verbessern und eine adaptierte Therapie einleiten zu können. Ergänzend stellt die diagnostische Laparoskopie ein probates Mittel zum Ausschluss bzw. zur Detektion fortgeschrittener respektive metastasierter Tumorstadien dar, wobei hier der zusätzliche Nachweis von IFCCs helfen kann, das Rezidivrisiko zu evaluieren und ggf. aggressivere Behandlungsstrategien zu planen.

\section{Korrespondenzadresse}

\section{Haeder}

Klinik für Allgemein- und Viszeralchirurgie, Schwerpunkt für endokrine und onkologische Chirurgie, Diakoniekrankenhaus Henriettenstiftung gGmbH, Marienstr. 72-90, 30171 Hannover Lars.Haeder@ddh-gruppe.de

Interessenkonflikt. Der korrespondierende Autor gibt für sich und seinen Koautor an, dass kein Interessenkonflikt besteht. 